\title{
The Manipulator Cleared the Surface of Fluid Pipelines
}

\author{
Bin $\mathrm{Li}^{1, \mathrm{a}}$, Jinping Hong ${ }^{2, \mathrm{~b}}$, Zhengfeng Cao ${ }^{3, \mathrm{c}}$ \\ 1,2,3Traffic \& Mechanical Engineering School, Shenyang Jianzhu University \\ abinli2010@sina.com, ${ }^{b}$ hong-1111@163.com, ${ }^{\mathrm{C} m i a o \_r f @ s i n a . c o m ~}$
}

Keywords: Manipulator, Modified asphalt, Fluid Pipelines, Photoelectric detection

\begin{abstract}
Manipulator cleared the surface of fluid pipelines is the necessary pipeline engineering construction equipment. Developing an advanced automatic robotic pipeline construction and making it industry can satisfy the needs of pipeline engineering in the country improve mechanization level and work efficiency and reduce labor intensity. According to the actual situation, the paper conform the principle of the robotic work. Analysis the work process of the manipulator, the design gives the optical detection system. This manipulator adopts automatic rotary and feeding, intelligent control and automatic detecting effects and feedback. The movement of the manipulator can been regulated and controlled timely by the photoelectric detection device of the manipulator. It is suitable for cleaning the surface of various pipelines in the complex condition field. This paper studies the revolution, feeding and feedback of the pipeline construction of the manipulator. Finally, study and design a stable running, flexible movement, accurate positioning, the effect is automatically detected and reliable intelligent robot which compatible with the subject. This manipulator can well meet the requirements of operation.
\end{abstract}

\section{Introduction}

In the process of repairing the cracking and perforation of the liquid pipelines, it is need to remove the modified asphalt of the pipeline surface under the conditions of the liquid is still moving in the pipeline and then the damaged parts can be repaired. In China, such work is done by artificial with angle grinder. But in other countries, the modified asphalt on the pipeline surface is cleaned away mainly with high temperature and high pressure gas.

Shenyang Liaohai oil transport equipment manufacturing factory according to the characteristics of pipeline construction method in our country, proposed the idea of clearing the pipeline surface automatically in the wild and to strengthen the quality of the construction management.

\section{The principle of robotic work}

This manipulator is represented by the control system, photoelectric detection systems and mechanical components. The mechanical parts including gear ring pipe section, active driving part, driven motor driver section, light poles, screw and work device parts. This paper studies the revolution, feeding and feedback of the pipeline construction of the manipulator. It is realized an important part of the manipulator work automatically.

Manipulator institutions analysis. Gear ring rail 2 and gear ring rail 8 as guide line foundation is decorated in fluid pipelines 1 . Active drive system 3 is arranged on gear ring rail 2. Active drive system 3 drive driven drive system 7 by light poles 4 , which is arranged on another gear ring rail 8 . The output terminal of driven drive system 7 drive screw 6 , the other end of the screw 6 support to the box of the drive system 3 . The work device parts 5 namely angle abrasive machine equipments is installed on the screw 6 and the light bar 4,which are parallel to each other. Screw 6 and light poles 4 implement circular motion along the gear ring rail 2 and 8 what is driven by active drive system 3 and driven drive system 7 and it achieve an axial direction along the pan pipes which is driven by the screw 6 . 
The gear ring rail 2 and 8 are the basis of the movement of active drive system 3 and driven drive system 7 . The gear ring rail is comprised by gear parts, rail parts, supports feet connections and supporting feet. Integral structure consists of two semicircle structure composition, and the two parts are connected by high-strength bolts. There are dowel pin on the rail parts and it is convenient for location and installation. Gear ring rail position with pipeline through three supporting feet. There are two types of supporting feet in order to adapt to the need of corresponding pipe diameter.

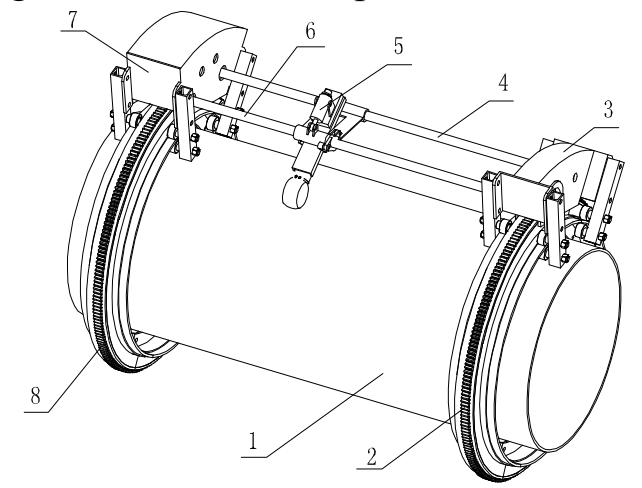

1 the fluid pipeline 2,8 the gear ring rail 3 active drive system 4 the light bar 5 the work device part 6 the screw 7 the driven drive system

Fig. 1. Overall diagram of the manipulator

Active drive system and driven drive system 7 adopts four hits leg with wheeled to support and guide. It can guarantee the active and driven box can move along very well with the gear ring rail orbits. There are driven drive gear in the active drive box. Driven driving gear axle is output shaft for transmission, and the hole within shaft is internal spline. The follower output drive shaft of active driving part drive light poles. The other end of the light bars drives the gear of the driven drive part. The drive gear of the driven part moves along the driven big gear rolling orbits driven by the light bars. This ensures that the active and driven two parts can be well synchronization operation.

The work device part is formed by a sliding frame, an angle grinder, a grinder bracket, a force composed of springs and counterweights etc. The nut on the sliding frame is double buckle close nut that can be separated. We can get the nut solved in the disassembly process or when we need to shift it and then the carriage be pushed along the screw and light poles fast so that the angle abrasive machine reach the position that we need. This can save time and improve work efficiency. Work device equipped with a strength spring and a balance weight. When the working device moves to different parts of the pipeline, the strength spring and the balance weight can offset the influence of the self-respect of the work device on the angle grinder. It makes the contact force between the surface of grinder and the pipeline remains the same. This will enable the angle grinder on pipe surface modified asphalt removal remained stable.

Robot motion analysis. The two gear ring rail's diameter of the manipulator is $1130 \mathrm{~mm}$, the module is 5 and the number of its teeth is 226 . The small gear's number of teeth is 18 and its module is 5.So, the gear reduction ratio is:

$$
\mathrm{i}=226 / 18=12.556 \text {. }
$$

Select the gear ratio reducer $i=105.58$. The output speed of the reducer is $13 \mathrm{rpm}$. And the speed of the robot moved around the fluid pipelines is:

$$
\mathrm{n}_{2}=\mathrm{n}_{1} / \mathrm{i}=13 / 12.556=1 \mathrm{rpm} \text {. }
$$




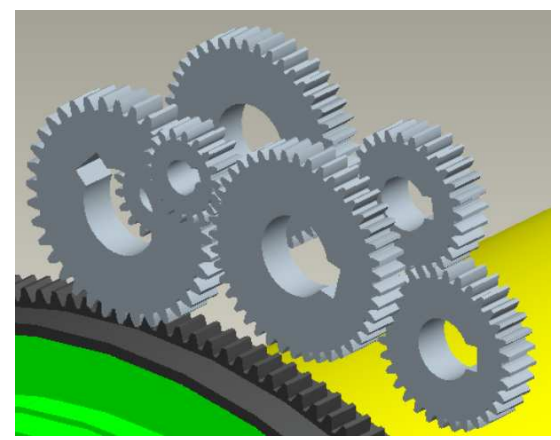

Fig. 2 Schematic diagram of the driven gear box

The relationship between the gears in the driven part is shown in figure 2.All the gear reduction ratio is 6.22 . The gear reduction ratio of the drive part is 2 . The gear reduction ratio from the output of the reducer to the output of the driven part is 12.44 . This can guarantee that when the driving system move one round, the screw turn a circle to achieve a pitch shift. The nominal diameter of the screw is $\varnothing 40$ and its pitch is $10 \mathrm{~mm}$. That is to say when the work device part move a trip around the pipe, it translates along the pipe axis shift $10 \mathrm{~mm}$. Choose the diameter of the steel brush wheel is $120 \mathrm{~mm}$, the width is $12 \mathrm{~mm}$, the amount of feed is $10 \mathrm{~mm} / \mathrm{r}$ and lap between upper is $2 \mathrm{~mm}$.

\section{Detection of residues in the working process}

When the robot is cleaning the asphalt on the surface of the pipe, the residue on the surface of the pipeline is judged mainly by the human eye. This is not conducive to the robot's automation and intelligent. This manipulator adopts photoelectric detection technology, the effect of the work of the manipulator real-time monitoring. And the movement of the manipulator can been regulated and controlled timely. Make the manipulator clear the pipe surface again where failed to meet the requirement and this can improve the working efficiency and the construction quality of the manipulator.

When the light shines on the opaque surface, it will produce diffuse reflection, refraction, absorption, etc. And there will also be mirror reflection light. According to the surface reflection of light, objects can be divided into smooth, semi-smooth and non smooth objects. When the measured surface is smooth, using the general illumination method can complete the task. The main reason is: the mirror reflected light of smooth metal surface is very strong.

The photoelectric detection device of the manipulator uses ordinary light source with certain angles to illuminate the pipe surface being processed. Incident light absorbed and reflected by the pipe surface, and then reflected into the receiving device. The light intensity of the light source is constant. When the pipeline surface is not very smooth with remains, the pipe surface happened to scattering, and the absorption of incident light is very strong and the reflection of incident light is not very good. The result is the light intensity of the reflected light into the receiving device is weak. When there is no residue remaining on the pipeline surface, the level of the pipe surface removal reach to construction requirements, the pipeline surface is smooth; the absorption of incident light is very weak, the reflection of incident light is good. The result is the light intensity of the reflected light into the receiving device is strong. If the pipeline surface is very smooth, there will be specular reflection, and then the intensity of the reflected light into the receiving device is stronger. 


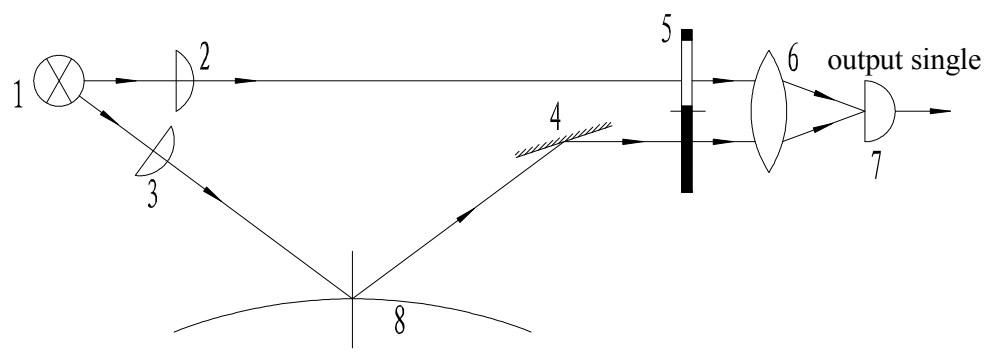

Fig. 3. Principle diagram of the photoelectric detection

A simple detector and basic detection methods can compose a simple optical detection system. A light produced by the light source 1 as a single light become parallel beams through the lens 3 . It illuminates the surface of the pipeline 8 and was reflected. The light reflected by the reflecting mirror 4 and focused on the photoelectric detector 7 by the convex lens 6 . The other light as the reference light through the lens 2 and the convex lens 6 reach the photoelectric detector 7 . The rotating modulation disc 5 has uniform distribution round holes, so that the single light and reference light can illuminate alternately on the photoelectric detector 7 . As the incident light in the signal light path is scattered, absorbed and reflected, so that the light intensity of the light illuminated to the photoelectric detected is changed. The result is the output signal of the photoelectric detected is square wave signal. The square wave amplitude is proportional to the difference between the signal light intensity and reference light intensity. That is also proportional to the number of remaining residue on the surface of the fluid pipes. The residue left on the fluid pipeline surface is more and the square wave amplitude is greater. On the contrary, the pipe surface residue remaining less, that the pipeline surface is smoother, the square wave amplitude is smaller. This is in order to effectively eliminate the impact of the strength fluctuation of the light source and the light background, using differential principle, design the differential compensation sensor with single light source.

\section{Conclusions}

This paper studies the revolution, feeding and feedback of the pipeline construction of the manipulator. It is realized an important part of the manipulator work automatically. Establish the drive method and the work principal of the manipulator and analysis the mechanical properties of the manipulator. This lays the foundation for designing the whole machine which has the best operating characteristics.

\section{Acknowledge}

This study was financially supported by Shenyang Science and Technology Project (1063290-1-002) and Liaoning Natural science fund Project (20102185).

\section{References}

[1] Min Jing, Photoelectric detection technology applications in the thickness measurement, science \& technology information. 33 (2009) 73 74.

[2] Zhaobang Pu, Photoelectric test technology, China Machine Press,Beijing,2004.

[3] Xinghua Qu, Ying He, Feng Han, Xuhui Zhao, Shenghua Ye, illumination system for detecting random defects on strongly reflective and complex surfaces, acta optica sinica. 23(5) (2003).

[4] Ying Wang, Jianlin Wang, optoelectronic inspection of in-pipe surfaces, journal of applied optics, 29(5) (2008) 735 738.

[5] Enqi Wu, Yinglin Ke, Jiangxiong Li, photoelectric detection and 3D reconstruction system for the inner surface of small-diameter pipes, opto-electronic engineering, 34(1) (2007) 59 64. 